\title{
Charge state and time resolved plasma composition of a pulsed zirconium arc in a nitrogen environment
}

\author{
Johanna Rosén \\ Materials Chemistry, RWTH-Aachen, D-52056 Aachen, Germany \\ André Anders \\ Lawrence Berkeley National Laboratory, Berkeley, California 94720, USA \\ Lars Hultman \\ Department of Physics, IFM, Linköping University, Linköping SE-58183, Sweden \\ Jochen M. Schneider \\ Materials Chemistry, RWTH-Aachen, D-52056 Aachen, Germany
}

\begin{abstract}
The species and ion charge state evolution of a pulsed cathodic arc plasma was investigated at different pressures. A zirconium cathode was operated in a nitrogen environment, and the plasma composition was analysed by time-of-flight charge-tomass spectrometry. Large plasma chemistry changes were detected with respect to time and pressure. The $250 \mu$ s plasma pulse can be divided in two characteristic phases: a transient phase before $150 \mu$ s and a steady state phase for all later times. The measured changes in plasma chemistry in the transient phase at $p \sim 10^{-5}$ Torr are explained by charge transfer collisions, while the increasing $\mathrm{N}^{+}$fraction in the $p>10^{-5}$ Torr range most likely originates from erosion of the nitrided cathode surface. In the steady state phase, a pressure-induced change from higher to lower charge states was observed, which is mainly due to scattering of self-sputtered metal followed by metal ion-atom charge exchange collisions. These results are of importance for
\end{abstract}


understanding the evolution of thin film composition and microstructure during reactive plasma deposition.

\section{INTRODUCTION}

A cathodic arc is a discharge process generating highly ionised metal plasma, which can be used for thin film synthesis, ${ }^{1}$ ion implantation ${ }^{2}$ and ion injection into accelerators. ${ }^{3}$ The plasma originates from micrometer-sized cathode spots, which erode the cathode surface material during plasma generation. The evolving cathode surface chemistry is therefore decisive for the plasma composition, which in turn determines composition of a growing film in arc plasma deposition.

In the presence of residual as well as intentionally introduced gas, the plasma chemistry and ion charge states can be affected through changes of the cathode surface chemistry, ${ }^{4-7}$ and through interaction between the expanding plasma and the surrounding gas. ${ }^{8-11}$ The plasma chemistry is important for the evolution of the chemical composition of a growing thin film, while the energy of particles affects film density and texture. ${ }^{12}$ The ion kinetic energy is proportional to the charge state if the substrate is biased, which is often the case.

The influence of a reactive gas on the plasma composition and average charge state has been investigated. For example, Oks et al. ${ }^{8}$ showed reduced Al ion charge states with increasing oxygen pressure, though no gas ions were detected. Spädtke et al. ${ }^{13}$ reported similar findings on $\mathrm{Mo}, \mathrm{Fe}$ and $\mathrm{Ti}$ in a nitrogen background. Martin et al. ${ }^{14}$ investigated the photon and ion emission from a titanium cathodic arc plasma at varying nitrogen pressure, and Demidenko et al. ${ }^{15}$ and Sakaki et al. ${ }^{16}$ analysed the 
influence of pressure on the ionisation and excitation mechanism of $\mathrm{Ti}$ and $\mathrm{Mo}$, and $\mathrm{Ti}$ arc plasma, respectively. Ionisation phenomena and ion current signal were also investigated by $\mathrm{Kimblin}^{17}$ for varying cathode materials and different gases. The results of these investigators ${ }^{8,13-17}$ show a decrease of higher charge states and ion current signal with increasing pressure, and an increase in gas ion fraction. This was explained primarily by charge exchange reactions between metal plasma ions and surrounding gas atoms or molecules, but also by electron impact ionisation of gas.

The data available describing the temporal development of cathodic arc plasma is very limited. Anders and Jüttner ${ }^{18}$ observed a temporal change of the plasma composition in ion energy measurements, and further work focused on the evolution of ion charge states for the high vacuum case. ${ }^{19}$ Schneider et al. ${ }^{6}$ reported on the temporal development of a pulsed Al plasma stream at varying oxygen pressure, and Rosén et al. ${ }^{7}$ described the time and pressure dependence of $\mathrm{Zr}$ and $\mathrm{Cr}$ arc plasmas in nitrogen environment. The strong temporal dependence of the plasma chemistry $^{6-7}$ with non-metal ions in the beginning of the pulse was explained by the formation and erosion of a compound layer at the cathode surface.

No charge-state-resolved data on the temporal dependence of the plasma chemistry could be found in literature. However, such data is required since the energy gain in the sheath in front of the substrate is proportional to the individual ion charge state. Therefore we have investigated the charge-state-resolved plasma composition, both with respect to its temporal development as well as the influence of surrounding pressure. The plasma composition was found to be a strong function of pressure and time into the discharge pulse. Different possible mechanisms responsible 
for the evolution were identified. These results are of importance to reactive plasma processing, especially to pulsed processes utilizing substrate bias, since varying plasma chemistry translates to difficulties of controlling the compositional as well as the microstructural evolution of the film.

\section{EXPERIMENTAL DETAILS}

The plasma was generated in a multi-cathode vacuum-arc ion source, "Mevva V", at Lawrence Berkeley National Laboratory. ${ }^{20}$ Arcs were triggered by a high voltage flashover across a ceramic tube between a trigger electrode and the front face of a zirconium cathode of $6.25 \mathrm{~mm}$ diameter, with resulting plasma pulses of $\sim 250 \mu \mathrm{s}$ duration. The arc current amplitude in the flat portion of the pulse was $200 \mathrm{~A}$, and the pulse repetition rate was $0.8 \mathrm{~Hz}$. The plasma composition was measured using a timeof-flight (TOF) charge-to-mass spectrometer, described in detail in Ref. 21. Ions were extracted from the plasma $0.14 \mathrm{~m}$ from the cathode with an extractor voltage of 30 $\mathrm{kV}$, forming an ion beam. A set of annular electrostatic TOF gate electrodes was pulsed, thereby a $200 \mathrm{~ns}$ sample of this beam was selected for analysis. The $200 \mathrm{~ns}$ slice of beam propagated to a magnetically suppressed Faraday cup, located $1.03 \mathrm{~m}$ from the gate. The plasma composition can be calculated from the measured ion current-time dependence, since the flight time of the different ions depend on their charge-to-mass ratio. The TOF system was differentially pumped, with the pressure in the cathodic arc zone about a factor 35 higher than in the TOF zone. It should be noted that with differential pumping and an extraction voltage of $30 \mathrm{kV}$, the probability for changes in the beam composition is very limited due to the very short beam transport times after extraction. 
To obtain temporal resolution, the beam "slice" was scanned through the arc pulse, as schematically shown in Fig. 1. Time-zero was chosen to be the moment when the first ions from the pulse reached the extraction system, corresponding to the beginning of the pulse in the detection system. A precision delay generator made it possible to scan the gate pulse in time steps of $5 \mu \mathrm{s}$ within the first $30 \mu \mathrm{s}$, followed by steps of $10 \mu$ s up to $100 \mu$ s after triggering, and finally steps of 25 and $50 \mu$ s for later times within the arc pulse.

The pressure dependence was investigated by introducing nitrogen near the cathode and collecting data at five different pressures, from $8 \cdot 10^{-7}$ Torr vacuum base pressure up to a partial pressure of nitrogen of $1 \cdot 10^{-4}$ Torr. The pressures reported refer to the pressure measured in the TOF zone, while the pressure in the discharge region was higher, as mentioned above. The ion charge data were collected in the form of averages: about 25 arc pulses gave stabilized, average values.

\section{EXPERIMENTAL RESULTS AND DISCUSSION}

The $250 \mu$ s plasma pulse can be divided into two phases, namely before and after about $150 \mu$ s into the pulse, where the latter represents approximately a steady state in the plasma chemistry evolution. The former will hereafter be referred to as the transient phase, characterised by extensive changes in the plasma chemistry, both in time and with pressure. Most of the discussion will therefore deal with the transient phase. 


\section{A Transient phase}

\section{A.I Low pressure, $p<\sim 10^{-5}$ Torr}

At low pressure, $p<10^{-5}$ Torr, the beginning of the pulse is dominated by $\mathrm{Zr}^{4+}$ ions (up to $\sim 20 \%$ within the first $50 \mu$ s) and $\mathrm{Zr}^{3+}$ (up to $\sim 45 \%$, highest level is in the time period $\sim 50-100 \mu \mathrm{s}$ ), see Figs. 2-3. The balance is given by $\mathrm{Zr}^{2+}, \mathrm{Zr}^{1+}, \mathrm{N}_{2}{ }^{+}$, and $\mathrm{N}^{+}$, see Figs. 4-7. Small traces of oxygen ions ${ }^{7}$ were also detected but are not further discussed here. Anders and Yushkov ${ }^{22}$ reported higher ion charge states and higher ion velocities at the beginning of each arc pulse. The higher ion velocities corresponded to more than twice the steady-state average ion kinetic energy. As long as the arc current was rising, the arc burning voltage was higher than in steady-state. These observations indicate that more energy is invested per plasma particle in the beginning of the arc pulse, and therefore available also for ionisation. As the plasma expands into the chamber, it can do so without suffering from many collisions with gas molecules at this low pressure.

\section{A.II Elevated pressure $p>\sim 10^{-5}$ Torr}

As the pressure increases to $10^{-5}$ Torr and higher, large changes in plasma composition were measured within the first $\sim 50 \mu$ s of the pulse: An abrupt decrease in the fraction of $\mathrm{Zr}^{4+}$ and a more gradual decrease of $\mathrm{Zr}^{3+}$, combined with an increase in $\mathrm{N}_{2}^{+}$, as seen in Fig. 6. This behaviour is consistent with an increased interaction between the expanding metal plasma and the surrounding gas through asymmetric charge transfer (reactions between unlike systems)

$$
\mathrm{Me}^{\mathrm{x}+}+\mathrm{N}_{2} \Rightarrow \mathrm{Me}^{(\mathrm{x}-1)^{+}}+\mathrm{N}_{2}^{+}
$$

where $\mathrm{Me}^{\mathrm{x}+}$ stands for the $\mathrm{x}$-fold charged metal ion. The nitrogen molecule may dissociate in these processes. Written as individual reactions, one should consider 


$$
\begin{aligned}
& \mathrm{Zr}^{+}+\mathrm{N}_{2} \Rightarrow \mathrm{Zr}+\mathrm{N}_{2}^{+} \\
& \mathrm{Zr}^{+}+\mathrm{N}_{2} \Rightarrow \mathrm{Zr}+\mathrm{N}^{+}+\mathrm{N}^{+} . \\
& \mathrm{Zr}^{2+}+\mathrm{N}_{2} \Rightarrow \mathrm{Zr}^{+}+\mathrm{N}_{2}^{+} \\
& \mathrm{Zr}^{2+}+\mathrm{N}_{2} \Rightarrow \mathrm{Zr}^{+}+\mathrm{N}^{+}+\mathrm{N}^{+} \\
& \mathrm{Zr}^{3+}+\mathrm{N}_{2} \Rightarrow \mathrm{Zr}^{2+}+\mathrm{N}_{2}^{+} \\
& \mathrm{Zr}^{3+}+\mathrm{N}_{2} \Rightarrow \mathrm{Zr}^{2+}+\mathrm{N}^{+}+\mathrm{N}^{+} \\
& \mathrm{Zr}^{4+}+\mathrm{N}_{2} \Rightarrow \mathrm{Zr}^{3+}+\mathrm{N}_{2}^{+} \\
& \mathrm{Zr}^{4+}+\mathrm{N}_{2} \Rightarrow \mathrm{Zr}^{3+}+\mathrm{N}^{+}+\mathrm{N}^{+} .
\end{aligned}
$$

The cross sections for reactions of this type are vastly different because of the internal energy defect, $\Delta E$, which is the difference in potential energies (ionisation, excitation) between pre-collision and after-collision particles. If the energy defect is negative, $\Delta E<0$, the cross section is negligibly small. Reactions (2) - (5) and (7) have $\Delta E<0$. The remaining reactions are much more likely to occur, leading to a sharp reduction of $\mathrm{Zr}^{4+}$ and $\mathrm{Zr}^{3+}$ while increasing the fraction of lower zirconium charge states and forming nitrogen ions, in particular $\mathrm{N}_{2}^{+}$.

The theory of charge exchange involves a transient stage, as does three-bodyrecombination. When a multiply charged ion approaches an atom in a 'slow' collision, as is the case in our plasma, a transient, excited "molecule" is formed. An electron from the neutral target is state-selectively captured by the multiply charged ion in a certain distance from the neutral target atom, typically 0.3-0.6 nm. State selection is determined by a matching condition of the potential energy of the initial and final channel in a reaction window. While classical trajectory Monte Carlo (CTMC) method ${ }^{23,24}$ was found to be adequate in many instances, little is know about the specifics on zirconium charge exchange with nitrogen. 
With an increase in pressure above $\sim 3 \cdot 10^{-5}$ Torr, the fraction of $\mathrm{N}_{2}^{+}$slowly decreases (Fig. 6). Since the total number of ions detected in the same region (Fig. 8) is increased, the decrease is not in contradiction to a measured increase in the number of $\mathrm{N}_{2}{ }^{+}$ions (Fig. 9). An increase in the number of ions at about $30 \mu \mathrm{s}$ and $6 \cdot 10^{-5}$ Torr is detected with constant measured ion current. This is consistent with the proposed charge exchange mechanism, resulting in ion charge being distributed over more particles.

The presented variations in $\mathrm{Zr}$ ion and $\mathrm{N}_{2}{ }^{+}$ion fraction are accompanied by an increase in $\mathrm{N}^{+}$ion fraction, see Fig. 7, and increase in $\mathrm{N}^{+}$ion signal, see Fig. 10. The onset of the increase in ion current occurs at higher pressure for $\mathrm{N}^{+}$as compared to $\mathrm{N}_{2}{ }^{+}$. (Figs. 6-7 and 9-10 only show the first $100 \mu$ s in the pulse, since no changes after this time were detected.) The $\mathrm{N}^{+}$ions are here suggested to originate from a compound layer formed at the cathode surface. This argument is based on considering the cross-sections for the production of $\mathrm{N}^{+}$ions. The cross section of dissociative ionisation and charge transfer in collisions between metal ions and $\mathrm{N}_{2}$ is smaller than the cross section for production of $\mathrm{N}_{2}{ }^{+} \cdot{ }^{25}$ Collisions involving $\mathrm{N}_{2}{ }^{+}$ions are another possible source for $\mathrm{N}^{+}$, though the cross sections for the different reactions indicate a low probability for dissociation and formation of $\mathrm{N}^{+}$, as compared to collisional reactions that deplete the $\mathrm{N}^{+}$population. ${ }^{26,27}$

Bilek et al. ${ }^{9-10}$ and Chhowalla, ${ }^{28}$ suggested that the abundance of $\mathrm{N}^{+}$are due to dissociation of $\mathrm{N}_{2}{ }^{+}$, or dissociation of $\mathrm{N}_{2}$ by ion impact (dissociation energy $9.8 \mathrm{eV}$, Ref. 29) with the resulting $\mathrm{N}$-atoms ionized via additional collisions (ionization 
energy $14.5 \mathrm{eV}$, Ref. 30). However, in light of a much higher concentration of $\mathrm{N}_{2}$ as compared to $\mathrm{N}$ (from possible dissociation of $\mathrm{N}_{2}$ ), together with their similar ionization energy, this would necessitate a much higher $\mathrm{N}_{2}^{+}$than $\mathrm{N}^{+}$signal, which was not detected. Chhowalla also found $\mathrm{ZrN}^{\mathrm{n}+}$ ions, which was explained by cathode poisoning, ${ }^{28}$ i.e. the same cause above suggested to explain the existence of $\mathrm{N}^{+}$ions. A direct comparison between the experimental results is not possible due to large differences in the experimental setups.

More support for these suggestions for the origin of the nitrogen ions is found by comparing the measured $\mathrm{N}_{2}^{+}$and $\mathrm{N}^{+}$ion signals and calculated ion fractions. Starting with the fractions (Figs. 6-7), the peak of $\mathrm{N}^{+}$is found closer to the beginning of the pulse than the peak of $\mathrm{N}_{2}^{+}$. The $\mathrm{N}^{+}$fraction extends further into the pulse with increasing pressure, which indicates a thicker nitrided surface layer, or cathode compound layer shifting in composition towards higher nitrogen content. The extension of the $\mathrm{N}_{2}{ }^{+}$fraction into the pulse does not change up to the highest pressure range investigated. Turning to the measured signals of the two gas ion species (Figs. 9-10), a comparison shows smoother gradients for the $\mathrm{N}^{+}$signal, which is more focused towards the beginning of the pulse and higher pressures. Previously published results $^{6-7}$ suggested a compound layer on the cathode due to the presence of reactive gas ions. The results should be considered in light of these charge-state-resolved measurements. It is appreciated that the gas ion signal can be composed of both a molecular part and an atomic part, of which only the latter indicates the presence of a compound layer at the cathode surface. The increment of ion current towards the beginning of the pulse with increasing pressure is also indicative for the formation and erosion of a compound layer. The reason is a faster and more stable (less fluctuating) 
arc ignition/plasma formation process that reaches steady-state faster as the surface is increasingly nitrided. $^{18}$

In Figs. 9 and 10 there is a clear maximum in the ion signals around $5 \cdot 10^{-5}$ Torr, followed by a decrease as the pressure further increases towards $10^{-4}$ Torr. This reduction of ion signal is also seen in Fig. 8. It is an indication that a pressure level is reached at which scattering and recombination reduces the signal to the time-of-flight detector, which is consistent with literature data concerning the effect of pressure on the ion signal. ${ }^{4,17}$

In reactive plasma processing and thin film synthesis, the time and pressure dependence of the plasma composition is of importance concerning the ability to control the growth process and consequently the quality of the resulting film. Apart from the compositional evolution, the plasma affects the microstructure of the film through ion energy, which in turn has an impact on the nucleation density, adatom mobility and defect formation. The ion energy has been measured by Bilek et al. ${ }^{10}$ and Tarrant et al. ${ }^{31}$ and it has been found to be pressure dependent. The charge state resolved measurements reported here show both a strong time and pressure dependence of the ion charge states, indicating large variations in ion energy distribution during film deposition. Comparing pulsed and DC plasma production systems, the latter can be considered as operating continuously in the steady state phase, therefore showing less variation over time. Pulsed systems, going through the transient phase with every pulse, have broader variation of plasma composition and ion energy distributions, which may be acceptable or even advantageous but implies less ability to control film composition and structure. 
Charge exchange collisions with neutral metal atoms are usually neglected with the argument that the plasma is fully ionised, with the fraction of neutrals being very small. This is consistent with the finding ${ }^{32}$ that ion charge state distributions usually peak at charge state $2+$ or $3+$. However, recent research on a cathodic arc system similar to the present system identified an additional source of neutral metal atoms in the vacuum arc case, namely self-sputtering of metal in the energetic condensation process on walls and substrate. ${ }^{33}$ In essence, the very energetic ions produced in a cathodic arc discharge show a likelihood to sputter an atom from the surface they are condensing onto. Both kinetic and potential energy of ions contribute to a total energy supply per incident ion that significantly exceeds the typical surface binding energy. ${ }^{34}$ The sputtered atom is most likely a metal atom of the same kind, originating from previously condensed material. As with most sputtered atoms, a self-sputtered atom has a kinetic energy of typically a few eV, which translates in a characteristic time of $100 \mu \mathrm{s}$ for crossing the pre-surface volume of $\sim 0.1 \mathrm{~m}$ dimension. In light of these recent results, the increase of metal neutrals and their interaction with the fully ionised plasma flow from the cathodic arc source appears to contribute to the transitional phase $(t<150 \mu \mathrm{s})$ and the establishment of the quasicontinuous steady state phase $(t>150 \mu \mathrm{s})$.

While self-sputtered metal was identified to also be responsible (at least in part) for the temporal development of charge state distributions in the vacuum case, ${ }^{33}$ the effect is even more pronounced in the presence of gas. In the vacuum case, the density of sputtered metal atoms remained low in the pre-surface volume due to the 
relatively high energy $(\mathrm{eV})$. With increasing gas pressure, sputtered $\mathrm{Zr}$ atoms suffer collisions with gas atoms,

$$
\mathrm{Zr} \text { (sputtered, fast) }+\mathrm{N}_{2} \text { (slow) } \Rightarrow \mathrm{Zr} \text { (slower) }+\mathrm{N}_{2} \text { (faster), }
$$

which reduces their average velocity and enhances their density. This, in turn, increases the probability that metal ions from the cathode will make a charge exchange collision,

$$
\mathrm{Zr}^{\mathrm{x}+} \text { (plasma flow) }+\mathrm{Zr} \text { (sputtered, slowed) } \Rightarrow \mathrm{Zr}^{(\mathrm{x}-1)}+\mathrm{Zr}^{+} \text {, }
$$

which successively reduces the concentration of higher charged $\mathrm{Zr}$ ions while increasing concentrations of doubly and singly charged Zr ions (Figs. 2-5).

\section{B Steady state phase}

The steady state phase $(t>150 \mu \mathrm{s})$ is characterised by an almost constant total ion current signal, which is only slightly decreasing as the pressure increases (though, one should always keep in mind that averaged values are measured and discussed, while instantaneous values show rapid fluctuations due to the explosive nature of plasma production). At low pressures, $p<\sim 10^{-5}$ Torr, more than $40 \%$ of the plasma ions are $\mathrm{Zr}^{4+}$ and $\mathrm{Zr}^{3+}$. As the pressure is increased to $p>\sim 10^{-5}$ Torr, the particle concentrations of $\mathrm{Zr}^{4+}$ and $\mathrm{Zr}^{3+}$ gradually decrease and the populations of $\mathrm{Zr}^{2+}$ and $\mathrm{Zr}^{1+}$ increase, as shown in Figs. 2-5. In the steady state phase, the total fraction of nitrogen ions was less than $2 \%$ over the entire pressure range investigated.

The observed combination of 1) a strong pressure dependence of the metal charge states and 2) a comparatively low fraction of gas ions throughout the gas pressure range, is most likely due to the interaction of self-sputtered material with the 
background gas, followed by metal ion - atom charge exchange, as already explained for the transitional phase.

In addition, three-body-collisions, where electrons $\left(\mathrm{e}^{-}\right)$recombine with metal ions $\left(\mathrm{Me}^{\mathrm{x}+}\right)$ according to

$$
\mathrm{Me}^{\mathrm{x}+}+\mathrm{e}^{-}+\mathrm{B} \rightarrow \mathrm{Me}^{(\mathrm{x}-1)^{+}}+\mathrm{B}
$$

may also contribute, however, the probability for the two-body collisions exceeds the probability of three-body reactions. The recombination reaction (12) is most efficient with electrons as the third body. ${ }^{16}$ The corresponding three-body recombination rates are known to sensitively depend on the electron temperature, namely approximately as $\mathrm{T}^{-9 / 2}$ for electrons of a few eV. ${ }^{35,36}$ No quantitative information is available at this time on reduction of electron temperature due to interaction with background gas and ions. While the gas temperature is close to room temperature, ions cannot not be simply described by a temperature due to the high directed velocity of metal ions coming from cathode spots. ${ }^{37}$ The coupling of electrons with heavy particles via elastic collisions must therefore be considered as dependent on the species. The transfer of kinetic energy in a collision between an energetic electron (few eV) and a "cold" molecule is inefficient because its scales with the small factor $2 m_{e} / M$, Ref. 38 . The cross section for elastic collisions between electrons of a few eV energy and nitrogen molecules is about $1.5 \cdot 10^{-19} \mathrm{~m}^{-2}$, Ref. 39, exceeding inelastic cross sections by a large factor. Using the cross section, density estimates, and energy transfer factor, one can quickly see that electron cooling by elastic collisions with heavy particles is only effective at high pressure ( 0.1 Torr and higher), which is higher than the pressure estimated in our source region. These estimates indicate the limited effectiveness of electron cooling and three-body-recombination, and therefore the 
interaction of self-sputtered material with the gas molecules appears as the most important factor explaining the pressure dependence of the plasma composition for the steady state phase.

\section{CONCLUSIONS}

We have presented the first charge-state-resolved plasma composition analysis of an arc pulse $(\mathrm{Zr})$, as a function of both pressure $\left(\mathrm{N}_{2}\right)$ and time after arc ignition. The plasma chemistry was found to be a strong function of both time and pressure. This can be understood by considering formation of a compound layer on the cathode and interactions between the metal ions, the surrounding gas and the selfsputtered metal. In the transient phase $(\leq 150 \mu \mathrm{s})$ at low pressure, higher charge states are present in the beginning of each pulse. This result pertains to higher pressure $\left(\sim 10^{-}\right.$ ${ }^{5}$ Torr), but is less evident due to charge exchange collisions within the first $\sim 50 \mu \mathrm{s}$, between mainly $\mathrm{Zr}^{4+}, \mathrm{Zr}^{3+}$ and $\mathrm{N}_{2}$, resulting in increased fraction of $\mathrm{N}_{2}^{+}$. At elevated pressure, $p>10^{-5}$ Torr, the increasing fraction of $\mathrm{N}^{+}$ions most likely originates from erosion of the nitrided cathode surface. In the steady state phase of plasma formation ( $\geq 150 \mu \mathrm{s}$ ), a pressure-induced change from higher charge states to lower charge states is consistent with scattering of self-sputtered metal by the gas molecules, followed by charge exchange between metal ions and atoms, leading to a reduction of higher charge states and an increase of lower metal ion charge states when the gas pressure is enhanced. These results are of importance for understanding the compositional and microstructural evolution of thin films from pulsed cathodic arcs, through the plasma composition and through the resulting ion energy distribution. 


\section{ACKNOWLEDGMENTS}

This work was supported by the Alexander von Humboldt Foundation, the German Federal Ministry of Education and Research, Program for Investment in the Future; by the U.S. Department of Energy under Contract No. DE-AC03-76SF00098 at Lawrence Berkeley National Laboratory, and by the Swedish Foundation for Strategic Research (SSF) Materials Research Program on Low Temperature Thin Film Synthesis. 


\section{References:}

${ }^{1}$ J. M. Schneider, S. Rohde, W. D. Sproul, and A. Matthews, J. Phys. D 33, R173 (2000)

${ }^{2}$ I. G. Brown, Rev. Sci. Instrum. 65, 3061 (1994)

${ }^{3}$ H. Reich, P. Spädtke, and E. M. Oks, Rev. Sci. Instrum. 71, 1 (2000)

${ }^{4}$ D. R. Porto, C. W. Kimblin, and D. T. Tuma, J. Appl. Phys. 53, 4740 (1982)

${ }^{5}$ M. Kühn and F. Richter, Surf. Coat. Technol. 89, 16 (1997)

${ }^{6}$ J. M. Schneider, A. Anders, I. Brown, B. Hjörvarsson, and L. Hultman, Appl. Phys. Lett. 75, 612 (1999)

${ }^{7}$ J. Rosén, A. Anders, L. Hultman, and J. M. Schneider, J. Appl. Phys. 94, 1414 (2003)

${ }^{8}$ E. Oks and G. Yushkov, Proc. XVIIth International Symposium on Discharges and Electrical Insulation in Vacuum, Berkeley, 584 (1996)

${ }^{9}$ M. M. M. Bilek, M. Chhowalla, and W. I. Milne, Appl. Phys. Lett. 71, 1777 (1997)

${ }^{10}$ M. M. M. Bilek, P. J. Martin, and D. R. McKenzie, J. Appl. Phys. 83, 2965 (1998)

${ }^{11}$ A. Bugaev, V. Gushenets, A. Nikolaev, E. Oks, A. Anders, I. Brown, and G. Yushkov, $18^{\text {th }}$ Int. Symp. on Discharges and Electrical Insulation in Vacuum, Eindhoven (1998), pp.256-259.

${ }^{12}$ J. E. Greene, S. A. Barnett, J.-E. Sundgren, and A. Rocket, Ion Beam Assisted Thin Film Growth, edited by K. Itoh (Elsevier, Amsterdam, 1989), Chap. 5.

${ }^{13}$ P. Spädtke, H. Emig, B. H. Wolf, and E. Oks, Rev. Sci. Instrum. 65, 3113 (1994)

${ }^{14}$ P. J. Martin, D. R. McKenzie, R. P. Netterfield, P. Swift, S. W. Filipczuk, K. H. Müller, C. G. Pacey, and B. James, $14^{\text {th }}$ International Conference on Metallurgical Coatings, San Diego, USA (1987) 
${ }^{15}$ I. I. Demidenko, N. S. Lomino, V. D. Ovcharenko, and G. N. Polyakova, Sov. Phys. Tech. Phys. 29, 895 (1984)

${ }^{16}$ M. Sakaki, and T. Sakakibara, IEEE Trans. Plasma Sci. 22, 1049 (1994)

${ }^{17}$ C. W. Kimblin, J. Appl. Phys. 45, 5235 (1974)

${ }^{18}$ S. Anders and B. Jüttner, IEEE Trans. Plasma Sci. 19, 705 (1991)

${ }^{19}$ A. Anders, S. Anders, B. Jüttner, and I. G. Brown, IEEE Trans. Plasma Sci. PS-21, 305-311 (1993).

${ }^{20}$ I. G. Brown, Rev. Sci. Instrum. 65, 3061 (1994)

${ }^{21}$ I. G. Brown, J. E. Galvin, R. A. MacGill, and R. T. Wright, Rev. Sci. Instrum. 58, $1589(1987)$

${ }^{22}$ A. Anders, and G. Y. Yushkov, J. Appl. Phys. 91, 4824 (2002)

${ }^{23}$ D. S. Fisher, S. R. Lundeen, C. W. Fehrenbach, and B. D. DePaola, Phys. Rev. A, 63, $52712(2001)$

${ }^{24}$ A. Kumar and B. C. Saha, J. Phys. B, 31, L937 (1998)

${ }^{25}$ J. B. Hasted, Physics of Atomic Collisions (Butterworths, London, 1972)

${ }^{26}$ A. V. Phelps, J. Phys. Chem. Ref. Data, 20, 557 (1991)

${ }^{27}$ W. Freysinger, F. A. Khan, P. B. Armentrout, P.Tosi, O. Dmitriev, and D. Bassi, J. Chem. Phys. 101, 3688 (1994)

${ }^{28}$ M. Chhowalla, Appl. Phys. Lett. 83, 1542 (2003)

${ }^{29}$ A. G. Gaydon, Dissociation Energies and Spectra of Diatomic Molecules (Chapman and Hall Ltd, London 1968)

${ }^{30}$ Handbook of Chemistry and Physics, $72^{\text {nd }}$ edition, (CRC Press, Boston, 1991)

${ }^{31}$ R. N. Tarrant, M. M. M. Bilek, T. W. H. Oates, J. Pigott, D. R. McKenzie, Surf. Coat Technol. 156, 110 (2002)

${ }^{32}$ A. Anders, Phys. Rev. E, 55, 969 (1997) 
${ }^{33}$ A. Anders, to be submitted to Appl. Phys. Lett.

${ }^{34}$ A. Anders, Appl. Phys. Lett. 80, 1100 (2002)

${ }^{35}$ L. M. Biberman, V. S. Vorobev, and I. T. Yakubov, Kinetics of the Non-Equilibrium and Low-Temperature Plasma (in Russ.) (Nauka, Moscow, 1982)

${ }^{36}$ Yu. P. Raizer, Gas Discharge Physics (Springer-Verlag, Berlin, 1991)

${ }^{37}$ E. Byon and A. Anders, J. Appl. Phys. 93, 1899 (2003)

${ }^{38}$ M. A. Lieberman and A. J. Lichtenberg, Principles of Plasma Discharges and Materials Processing (John Wiley \& Sons, New York, 1994)

${ }^{39}$ I. P. Shkarovfsky, T. W. Johnston, and M. P. Bachynski, The Particle Kinetics of Plasmas (Addison-Wesley Publishing, Reading, MA, 1966) 


\section{Figure captions:}

Figure 1: Schematic describing the experimental procedure.

Figure 2: Concentration (particle\%) of $\mathrm{Zr}^{4+}$ ions vs nitrogen pressure (Torr) and time into arc pulse $(\mu \mathrm{s})$.

Figure 3: Concentration (particle\%) of $\mathrm{Zr}^{3+}$ ions vs nitrogen pressure (Torr) and time into arc pulse $(\mu \mathrm{s})$.

Figure 4: Concentration (particle\%) of $\mathrm{Zr}^{2+}$ ions vs nitrogen pressure (Torr) and time into arc pulse $(\mu \mathrm{s})$.

Figure 5: Concentration (particle\%) of $\mathrm{Zr}^{1+}$ ions vs nitrogen pressure (Torr) and time into arc pulse $(\mu s)$.

Figure 6: Concentration (particle\%) of $\mathrm{N}_{2}{ }^{+}$ions vs nitrogen pressure (Torr) and time into arc pulse $(\mu \mathrm{s})$.

Figure 7: Concentration (particle $\%$ ) of $\mathrm{N}^{+}$ions vs nitrogen pressure (Torr) and time into arc pulse $(\mu \mathrm{s})$.

Figure 8: Signal proportional to number of ions (all species) detected vs nitrogen pressure (Torr) and time into arc pulse ( $\mu \mathrm{s})$. 
LBNL-56277, appeared in the J. Applied Physics 96 (2004) 4793-4799

Figure 9: Signal proportional to number of $\mathrm{N}_{2}{ }^{+}$ions detected vs nitrogen pressure (Torr) and time into arc pulse $(\mu \mathrm{s})$.

Figure 10: Signal proportional to number of $\mathrm{N}^{+}$ions detected vs nitrogen pressure (Torr) and time into arc pulse $(\mu \mathrm{s})$. 
Figure 1, Rosén et al, JAP

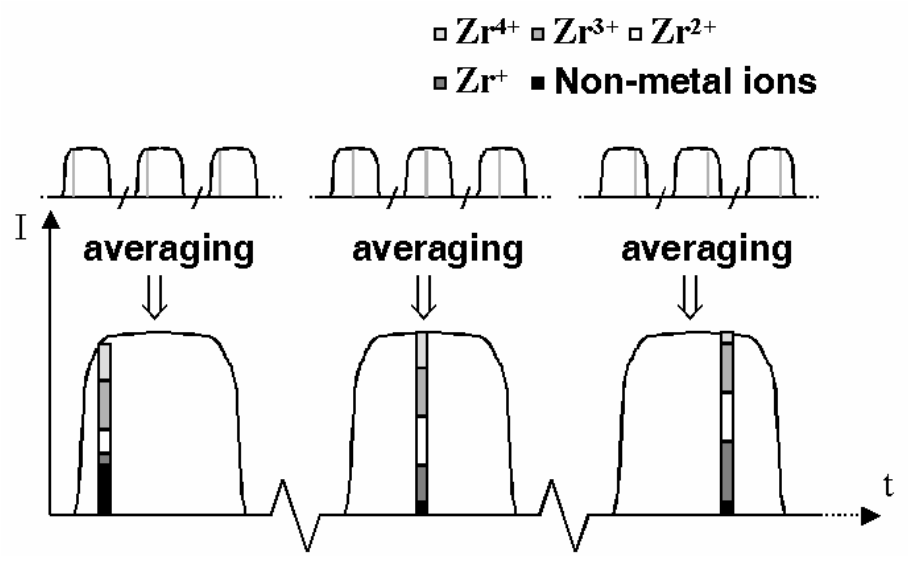


Figure 2, Rosén et al, JAP

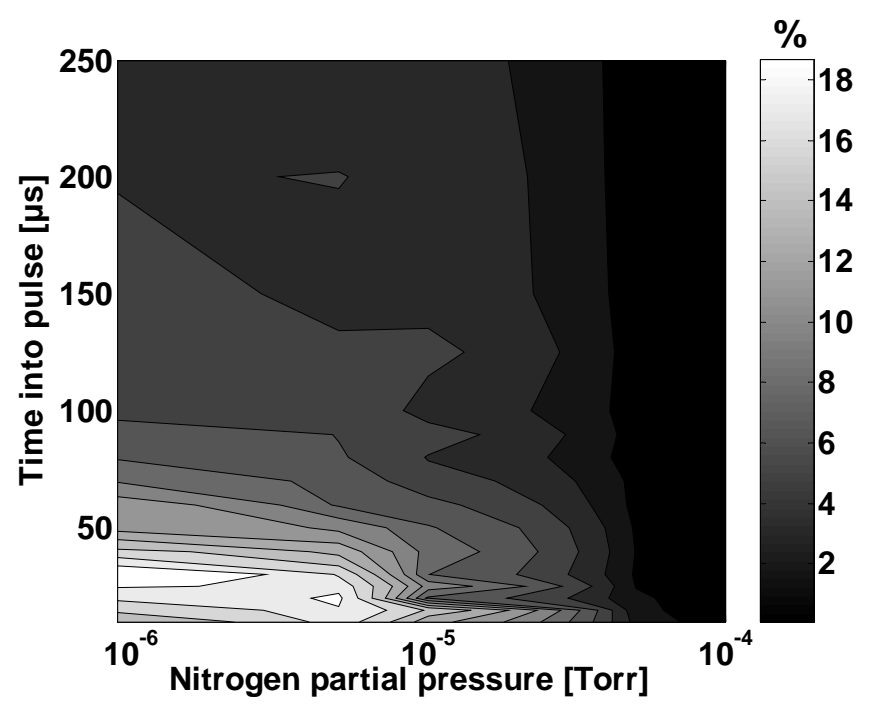


Figure 3, Rosén et al, JAP

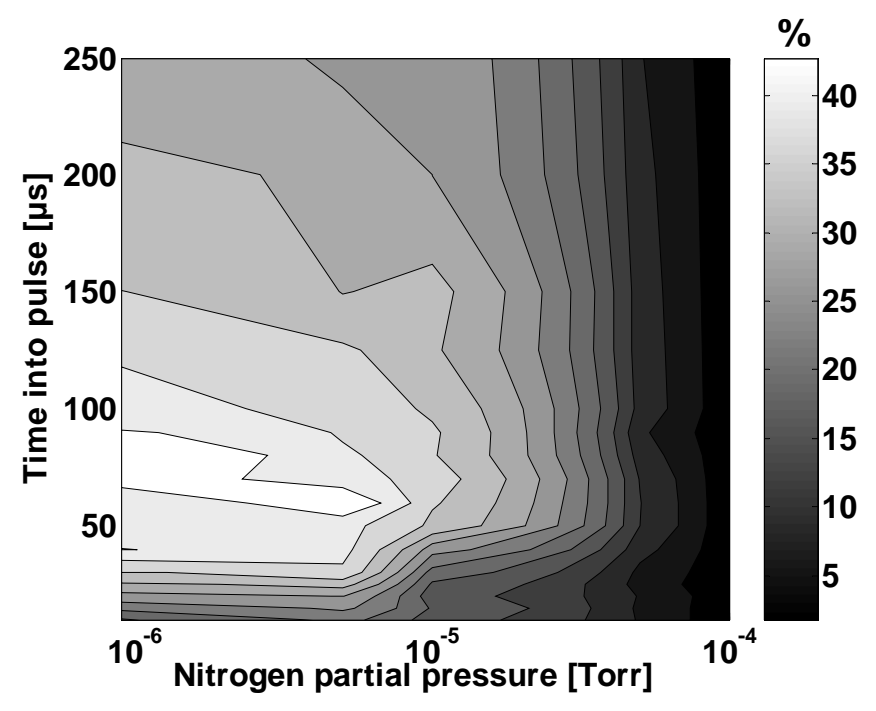


Figure 4, Rosén et al, JAP

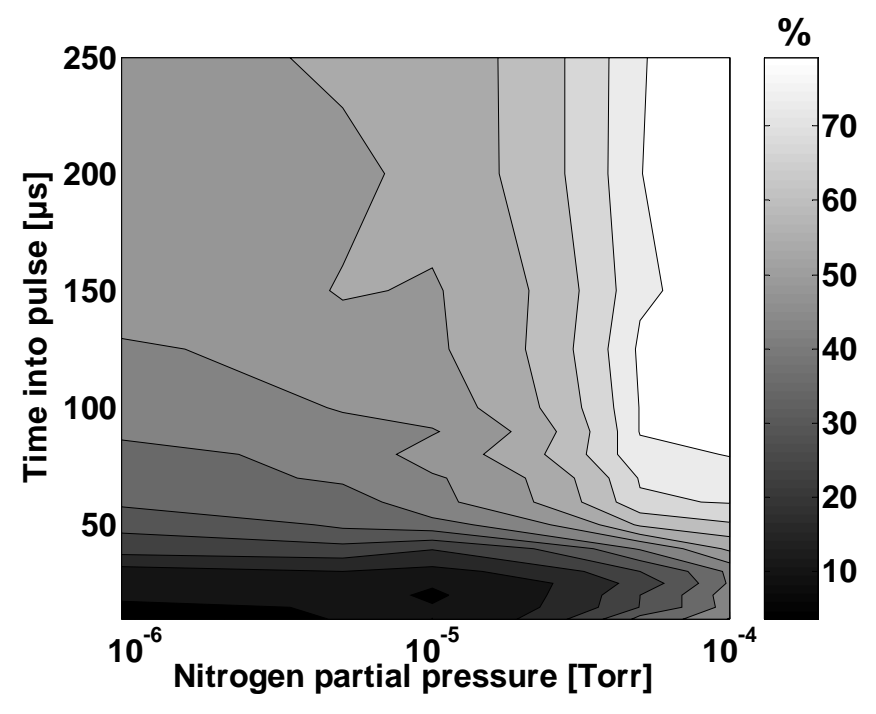


Figure 5, Rosén et al, JAP

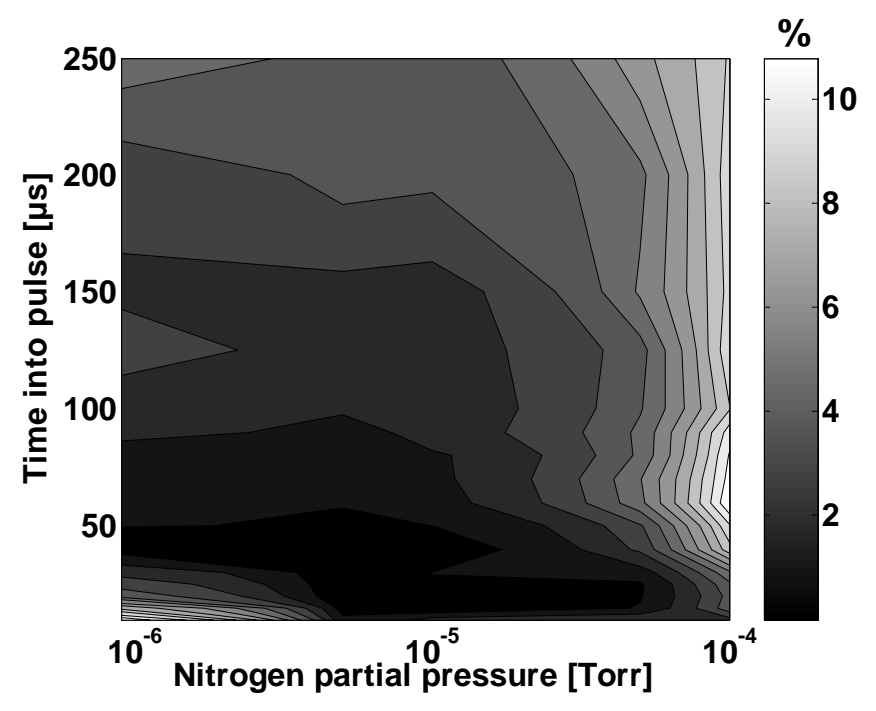


Figure 6, Rosén et al, JAP

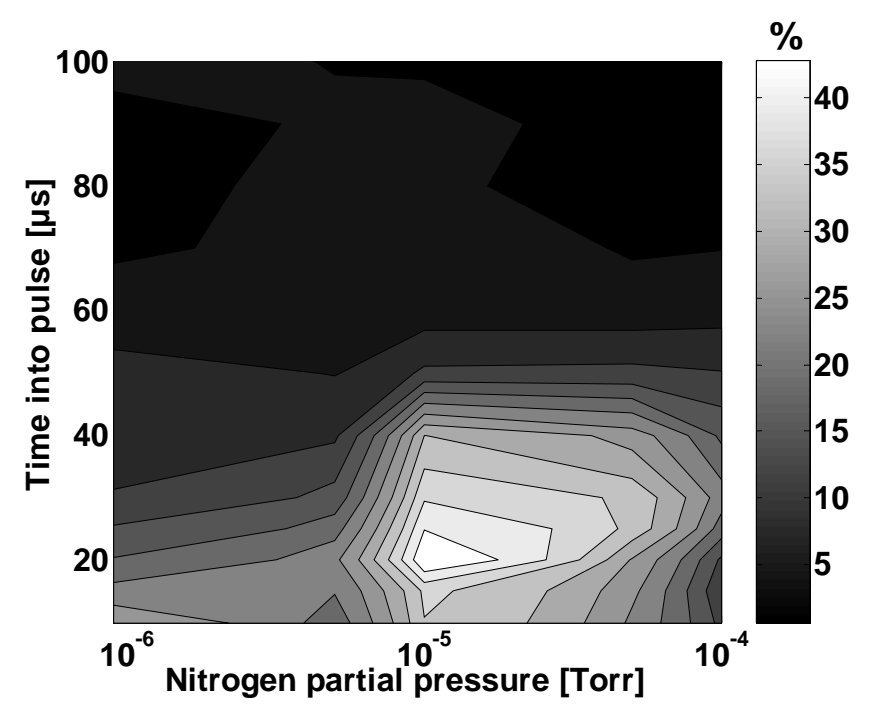


Figure 7, Rosén et al, JAP

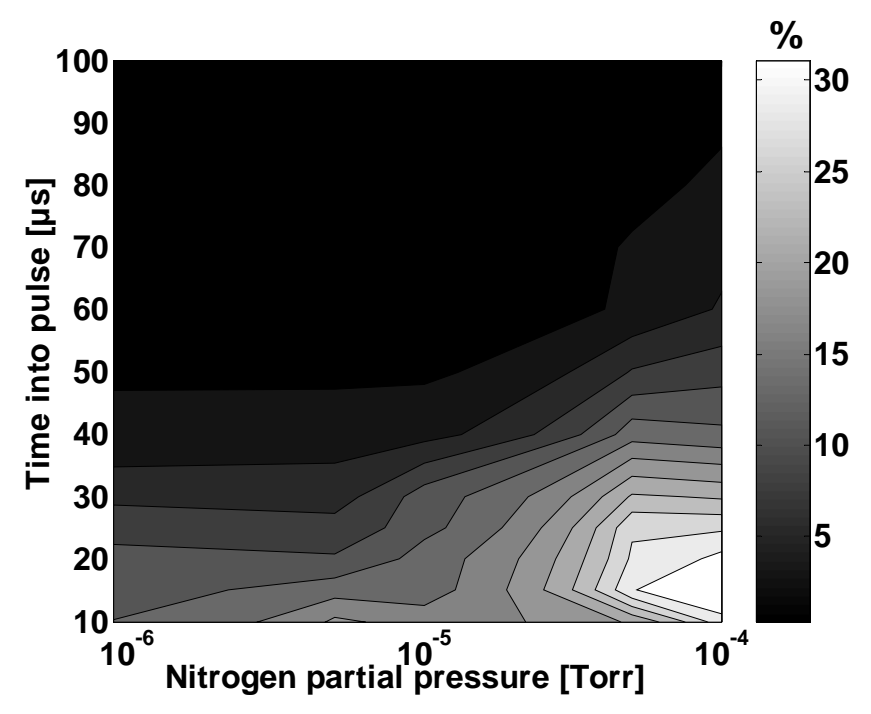


Figure 8, Rosén et al, JAP

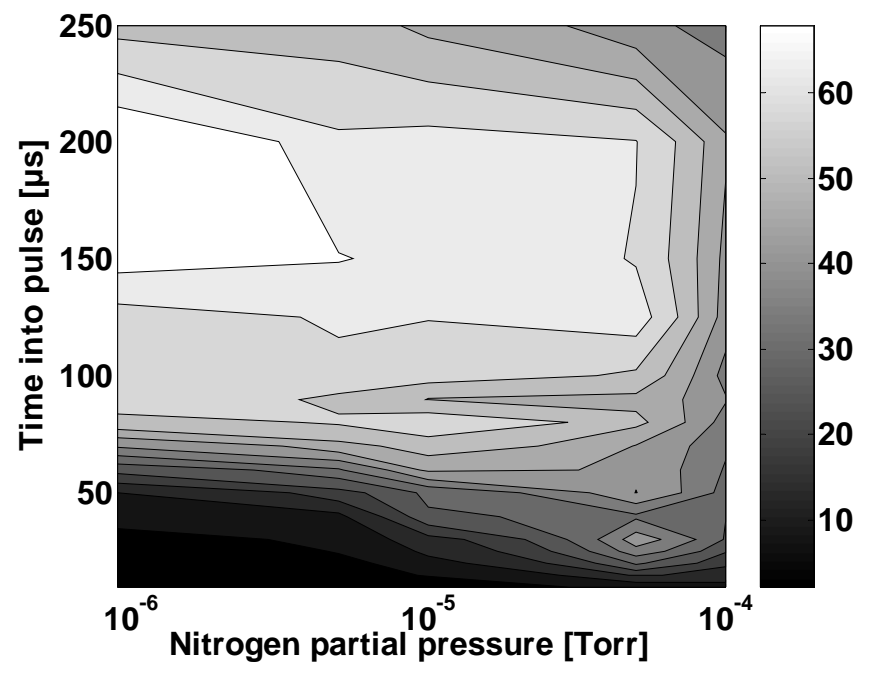


Figure 9, Rosén et al, JAP

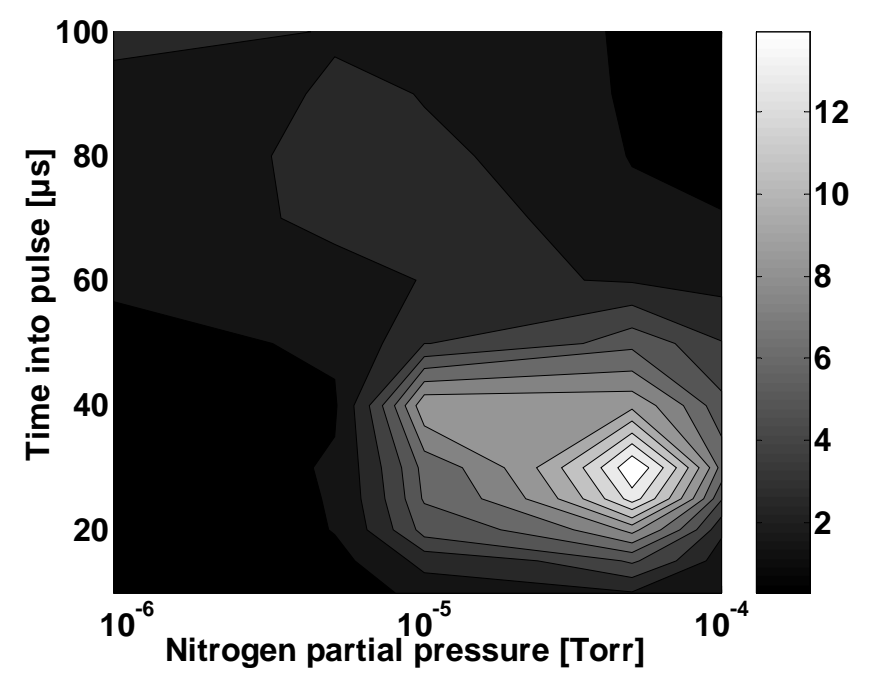


Figure 10, Rosén et al, JAP

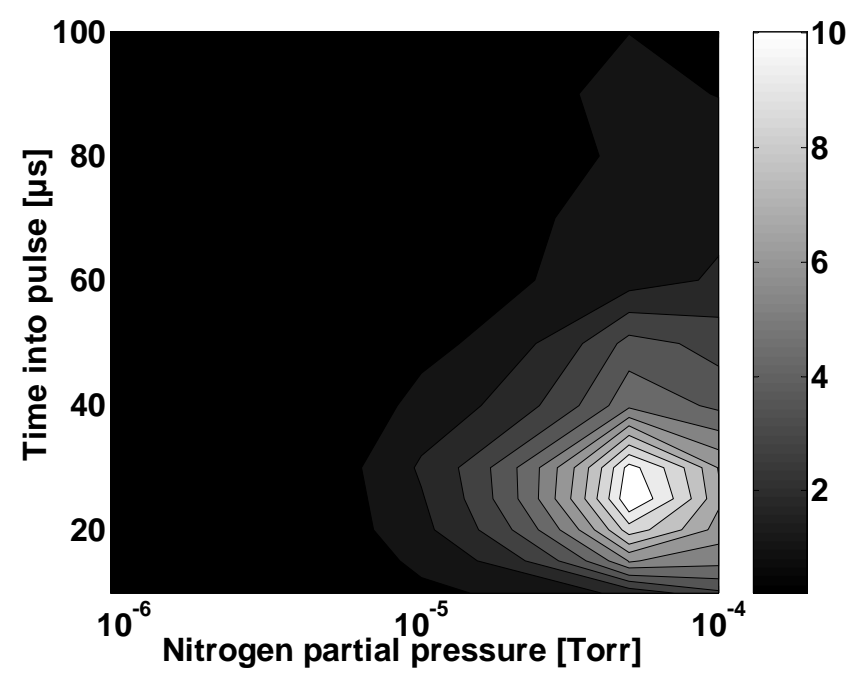

